\title{
MI VICO
}

\author{
Enrico Nuzzo \\ (Universidad de Salerno)
}

RESUMEN: La contribución presenta sintéticamente las líneas generales de la interpretación general de Vico que el Autor ha propuesto en una vasta producción en torno al filósofo napolitano y a la cultura de su tiempo. PALABRAS ClAVE: Vico, $350^{\circ}$ Aniversario, interpretación general, historización y actualización, E. Nuzzo.

My Vico

ABSTRACT: This contribution synthetically presents the main lines of the general interpretation of Vico that the Author has proposed in a vast production around the Neapolitan philosopher and the culture of his time.

KEYWORDS: Vico, $350^{\text {th }}$ Anniversary, general interpretation, historicity and update, E. Nuzzo.

\section{Il mio Vico}

RIASSUNTO: Il contributo presenta sinteticamente le linee principali dell'interpretazione generale di Vico che l'Autore ha proposto in una vasta produzione sul filosofo napoletano e sulla cultura del suo tempo.

PAROLE CHIAVE: Vico, $350^{\circ}$ anniversario, interpretazione generale, storicizzazione e attualizzazione, E. Nuzzo.

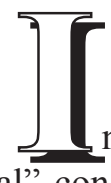

ncluso los estudiosos más lúcidos aprenden a convivir pronto "de forma natural" con los autores que han entrado más o menos establemente en su "vida intelectual" (mejor dicho, en tan absorbente parte de su vida).

Por lo que a mí se refiere, nunca he pacificado totalmente un sutil sentido de inquietud frente al elemento de arbitrariedad de la entrada en mi existencia de numerosos autores, problemas y argumentos que han cautivado mi interés (de los presocráticos y Platón a la metaforología contemporánea): antes que nada Vico, quien muy pronto se convirtió en mi "autor" (sobre el cual publiqué varios libros, además de alrededor de cincuenta ensayos). Sin embargo, cuando Pietro Piovani me

Este artículo responde a una invitación expresa por parte de la Dirección de la Revista para este volumen especial por el $350^{\circ}$ Aniversario del nacimiento de G. Vico, habiendo superado los criterios de valoración y del proceso de aceptación. 
preguntó si iba a aceptar el compromiso de escribir en pocos meses un pequeño volumen que saldría en una colección divulgadora, ${ }^{1}$ en realidad ya había dirigido hacia el siglo XVIII -y la historia de la "conciencia histórica"- mis intereses principales; desde luego, Vico debía parecer el camino obligado para quien, como yo, había manifestado desde muy joven una inclinación "natural" hacia un historicismo integral. Aquel personal «nascimento» a los estudios viquianos se daba en la temporada de su "nuevo curso", caracterizada antes de todo por una significativa reinserción de Vico en el contexto de su tiempo y signada por la iniciativa de Piovani de fundar el "Centro di Studi Vichiani" y, a la vez, su Bollettino. A partir de ese momento, mis estudios sobre Vico se han relacionado constantemente con investigaciones sobre el pensamiento moderno (la cultura de impronta cartesiana, el libertinismo, las tradiciones y los "lenguajes" del pensamiento político e histórico moderno, etc.) y la cultura y "tradición" napolitana, con libros (sobre Caloprese, Doria, Di Capua) ${ }^{2}$ y numerosos ensayos.

Este tipo de enfoque historiográfico "contextualizador" -que, en realidad, luego no ha tenido particular seguimiento- favoreció mi propensión personal hacia una escritura preliminar de tipo "ensayístico" que, a pesar de esto, no me alejó de la tarea de trazar una "interpretación general".

Voy a intentar exponer los puntos esenciales de esta interpretación, de la que parece emerger un Vico en su conjunto bastante insólito.

De acuerdo con una tendencia crítica ahora ya no tan exigua, por lo general "mi Vico" está colocado plenamente en la "modernidad". Él plantea perspectivas muy originales y fecundas en ámbitos problemáticos derivados de experiencias fundamentales de la filosofía moderna, sobre todo si se considera que la agenda teórica principal de la primera parte del Setecientos seguía dependiendo mucho de la especulación de la segunda del Seiscientos. Antes de todo desde el punto de vista epistemológico y gnoseológico, los adversarios en contra de los cuales se puso Vico eran Descartes y cierta cultura de impronta cartesiana. Sin embargo, los verdaderos "enemigos" del autor de la Scienza nuova eran Hobbes, Spinoza, Bayle, es decir, los mayores representantes de la orientación teórica más peligrosamente impía: aquella de la naturalización de cada orden, el físico y el humano histórico.

La respuesta extremadamente "moderna" de Vico, sobre todo al modelo spinoziano de un "orden inmanente", atañó al ámbito "humanológico-prácticocivil". A diferencia de una amplia directriz de estudios, el Vico que reconstruí es un pensador intrínsecamente "político", como pocos filósofos en la historia del pensa-

1. Fue mi Vico, Vallecchi, Florencia, 1974.

2. E. Nuzzo, «La tradizione filosofica meridionale», en Storia del Mezzogiorno, vol. X, tomo $3^{\circ}$, Edizioni del Sole, Nápoles, 1992, pp. 17-127; Verso la "Vita civile". Antropologia e politica nelle lezioni accademiche di Gregorio Caloprese e Paolo Mattia Doria, Guida, Nápoles, 1984; Vite e scritti di capitani. Attorno alla Vita di D. Andrea Cantelmo di Leonardo Di Capua, Alfredo Guida, Nápoles, 2005. 
miento. ${ }^{3}$ Con respecto a esto, él se puede considerar heredero de la tradición clásico-humanista de la "vida civil" y de los correspondientes intereses por los saberes de lo "verosímil", de la retórica y de la jurisprudencia. Por lo demás, en Vico fue muy exigua la herencia de la filosofía humanista-renacentista de marca platónica, inscrita como estaba en ontologías metafísicas de un orden total del ser, barridas por la especulación de tipo cartesiano.

Sin embargo, el discurso humanológico de Vico necesitaba de una fundación metafísico-teológica. En sustancia, su filosofía fue "sin naturaleza", pero no exenta de una orientación hacia una "antimoderna" metafísica de la naturaleza antimecanicista y antiatomista; pero, sobre todo, no sin "naturaleza metafísica", y de fundamento teológico: una metafísica cristiana pero exclusivamente interesada por la «salvación de las naciones» (una "metafísica-teología de la historia"), sin ningún interés por la salvación de las almas o hacia los espacios profundos de la interioridad.

Vico contestó a Spinoza desplazando a la historia la búsqueda del «orden», esto es, de un orden guiado por un principio providencial que solo actúa por "vías simplicísimas», sobre la base de una antigua tradición teológica y de acuerdo con el "galileano" principio moderno de simplicidad. De hecho, Vico teorizó un modelo de providencia para el que un Dios benéfico había instituido un orden que se origina de las vías todas «naturales» de las respuestas espontáneas de las disposiciones de la naturaleza humana a constantes y, al mismo tiempo, dinámicas formas de «necesidades» e «intereses». La Scienza nuova de las naciones quería ser la demostración y la narración de la producción de ese orden, demostración que se acompañaba con la función «práctica» de convertir a los sujetos de los tiempos más avanzados, en la conquista de lo verdadero y lo justo, en seres conscientes de sus responsabilidades ético-políticas. Esta posición de una Providencia "inmanente", "ortodoxa" pero no exenta de insidias graves, ha sido sometida a numerosas interpretaciones, que la han leído como heterodoxa, convirtiendo a Vico en un pensador sustancialmente no cristiano, spinozista o "pagano". En efecto, su modelo se originaba desde la cultura teológica cristiana, precisamente de la directriz de la potestas ordinata de Dios que, en la filosofía coeva de Vico, encontró su máxima y renovada expresión en la conceptualización de lo divino elaborada por el cartesiano Malebranche, de la que resultó notablemente influenciada la solución viquiana. ${ }^{4}$

3. Sobre esto cfr., sobre todo, mi volumen Tra religione e prudenza. La "filosofia pratica" di Giambattista Vico, Edizioni di Storia e Letteratura, Roma, 2007. Vid. también -entre otros- los ensayos recogidos en el volumen Il declino della quiete. Tra aristotelismo politico e ragion di Stato a Napoli dal primo Seicento a Vico, Edizioni di Storia e Letteratura, Roma, 2014.

4. A este propósito cfr. sobre todo mis ensayos: «Between Orthodoxy and Heterodoxy in Italian Culture in the Early 1700s: Giambattista Vico and Paolo Mattia Doria», en The Intellectual Consequences of Religious Heterodoxy 1650-1750, S. Mortimer y J. RoberTson (EDS.), Brill, Leiden-Boston, 2012, pp. 205-234; «Religione e "ortodossia" in Vico. I fondamenti teologici della razionalità storica», Bollettino del Centro di studi vichiani, XLVI, 2016, pp. 11-52; «Las "vías simplísimas" de la Providencia. Vico y el "principio de simplicidad" en la filosofía cristiana entre los siglos XVII y XVIII», Cuadernos sobre Vico, 30/31, 2016/2017, pp. 259-276; «Ai limiti dell'ortodossia cristiana. "Principio di semplicità" e ordine naturale e storico in Malebranche e Vico», en Malebranche e i suoi contemporanei, al cuidado de R. CARBONE, Laboratorio dell'ISPF, XIV, 2017, pp. 1-77. 
En mi opinión, en Vico, tanto la concepción de la providencia que se realiza por vías naturales, como la antropología tensional que con aquella se enlaza, tienen que ver con una perspectiva de "agustinismo temperado" que se afirmó en la modernidad con caracteres muy diferentes de los del agustinismo luterano. De hecho, el tema crucial del nexo de relación-transformación entre voluntades egoísticamente pasionales de los hombres, por un lado, y orden social inintencionalmente alcanzado, por el otro, se puede leer en la perspectiva de una reconstrucción de uno de los dos caminos principales a través de los cuales se dio el "autoagotamiento" del agustinismo en la modernidad: un "agustinismo temperado", abierto a tonos "dramáticos" pero no "trágicos". Así las cosas, en Vico la naturaleza humana -aunque «labefacta» por el pecado- posee en su interior el legado providencial de una conativa «vis veri» operante, de forma muy subterránea, en el conseguimiento de un dinámico orden humano.

La propuesta de reconducir vitales «necesidades» e «intereses» hacia la función de «ocasiones», por otra parte, prohíbe olvidar el papel de «forma» y «norma» de la mente humana, y el carácter de axiológica «eminencia» de sus funciones racionales. Sobre la base del decisivo criterio del «verum-certum» se instituye la contracción de lo verdadero y justo en la factualidad de aquellos fenómenos que, a su vez, son los únicos capaces de detenerlos en su tiempo (según la "lógica del por lo menos" de la que he hablado a menudo en mis escritos), de manera que una preeminencia factual de lo "prerreflexivo" caracteriza tanto la acción humana como los «progresos» del curso histórico. Sin embargo, esto no significa que "verdadero" y "justo" pierdan su "platónica" plenitud normativa, su "teleologicidad" y que, finalmente, se deben entregar sobre todo a la responsabilidad de los poderes de una mente «desplegada» («spiegata»).

Desde el punto de vista más estrechamente epistemológico-gnoseológico, Vico se medía - desde una perspectiva "pos-racionalista", no anti-racionalista- con las filosofías "modernas": tanto cuando elaboraba una visión mucho más abierta de las facultades cognoscitivas con la extraordinaria valorización de la fantasía y del ingenio, como y sobre todo cuando fundaba un acercamiento "demostrativo" a las verdades del "mundo histórico", basado antes que nada en la aplicación que se le hace de cortantes «pruebas filosóficas», respaldadas por lo que he llamado el «principio de lo imposible-que-no», esto es, la «verdad de razón». Es un principio propio de un pensamiento "genetizante" y, al mismo tiempo, capaz de construir un saber histórico absolutamente no conjetural, que confía inusitadamente en esenciales poderes demostrativos de la razón. ${ }^{5}$

En este primer plano nos hallamos ante el mismo pensador, empeñado en la fecundísima especulación sobre el fundarse, refundarse y darse del sujeto humano,

5. Remito en particular a páginas del volumen Tra ordine della storia e storicità. Saggi sui saperi della storia in Vico, Edizioni di Storia e Letteratura, Roma, 2001. 
esto es, el autor que ha profundizado como nadie antes en las facultades primigenias y productivas de aquel mundo originario, sobre el cual escribió páginas entre las más agudas e intensas de su admirable escritura.

De ahí que Vico haya propuesto una concepción globalmente "equilibrada" de la complejidad y productividad del conjunto de las facultades humanas, sin dejar de recurrir a las indispensables funciones de la "razón", pero rescatando aquellas más cargadas de "corporeidad" de tradiciones que las habían condenado a una subordinación ontológica, según un "principio de integración-contracción" que halla su expresión intrínseca en una singular y esencial conceptualización oximórica (como en la imagen de la «sabiduría poética» en la edad de los «héroes campesinos»...). Así las cosas, cabe guardarse de interpretaciones (cuya fortuna parece haber disminuido respecto de hace algunas décadas) que curvan todo Vico hacia la figura del diagnóstico de los males de la razón y hacia el autor de una concepción para la cual no solo se identifica una autónoma y productiva "lógica de la fantasía", sino que esta se erige en superior paradigma especulativo de un pensamiento radicalmente alternativo con respecto a la "razón moderna" o, en general, al dominio del lógos. Además, una cosa es el reconocimiento de la contribución a la individuación de una específica "lógica" del pensamiento "lógico-mítico", y otra cosa es la tergiversación de una hermenéutica del mito en realidad empeñada en la operación -no exenta de sabor "racionalista"- de aclarar sus contenidos determinados (como la experiencia "estructural" de todos los Hércules que se repiten en todas las naciones).

Por otro lado, cabe subrayar que los elementos más fértiles de originalidad -los llamaríamos de "excedencia especulativa"- de la meditación viquiana se hallan en este Vico. Dentro de las máximas expresiones de esta gran energía teórica, junto con la apertura hacia el mundo de la «sabiduría poética», están las ideas sobre el lenguaje, al trazar una fundación teórica del sujeto humano en clave "lingüística" o "sematológica". Aunque la idea de similar fundación de la intersubjetividad humana lleva siendo desde hace tiempo "metabolizada" en el pensamiento contemporáneo, regresar a Vico en lo que a esto se refiere puede significar retomar sus huellas especulativas no agotadas. Está claro que se puede correr el riesgo de sobreexponer la reflexión viquiana a preguntas más bien "nuestras", pero parece indiscutible que esta acoge aperturas muy agudas sobre la "genealogía de lo humano". Finalmente, el mismo Vico parece dejar de lado el punto central, es decir, que el nacimiento de lo humano es realmente su "re-nacimiento", adelantado por una "historia metafísica" en la cual una secreta «vis veri» ha permitido al bestión desmedido detener su divagación (en efecto, no hay vacío abismal ni antes de la re-emersión de lo humano, ni después del eventual naufragio de las naciones). De todas formas, tal "genealogía" impulsa una mirada inquieta hacia el tema de la distancia de nosotros de la condición de lo "corpóreo", de lo "pático": en lo absolutamente no-humano, así como en el originario humano casi totalmente pático, que solo fatigosamente se 
puede intentar no «imaginar», sino «comprender», y decir de forma narrativa; tal como en la experiencia de pérdida que se da al pasar a la lengua abstracta, frente a la escisión entre palabra y cosa. Al mismo tiempo se debe recalcar que a Vico no se puede atribuir una sensibilidad especulativa contemporánea por una acción hermenéutica originaria del significar, y que la pérdida de lo pático en la abstracción de la lengua no lo lleva a una dolorida meditación sobre la llegada de una distancia ontológica. Lo humano primigenio impregnado de afectividad pática, aunque se halla en la fenomenología de lo humano, sigue siendo lo que procura «horror» a «nuestras civilizadas naturalezas» («nostre ingentilite nature»), propias de una humanidad que ha conquistado un mayor acceso a lo «verdadero-justo».

He afirmado varias veces que la aproximación "genética" e "historicizadora" de Vico a todas las formas del mundo humano histórico permite reflexionar de forma problemática sobre el carácter "historicista" de su pensamiento: pero sobre la fundación de una configuración de "historicismo" diferente no solo -claro está- del "historicismo absoluto", sino también de aquel individualizador "crítico-problemático", porque concentra el interés en la génesis y en la producción, más bien, de las constantes "estructuras históricas" de lo humano. En ese cuadro, en el escenario de los progresos del siglo XVIII de la conciencia histórica moderna, se sitúa el inédito diseño viquiano de una historia "entera" y "progresiva" de la humanidad y de la civilización. Similar diseño - expresado ampliamente de forma narrativa- está sustentado por unos sólidos cimientos epistémicos y por una concepción del tiempo compleja. En particular, opino que en esta prevalece una cifra "progresiva" del devenir de las formas humanas, aunque se den diferentes imaginarios de sus sucesos temporales: el "ascendente" de la verdad y justicia (y de las instituciones jurídicas y políticas); el "parabólico" de las costumbres; el "simétricamente alternativo" de las formas del sistema de las facultades. Esto implica que es mucho lo que, constitutiva y dramáticamente, se pierde precisamente en los «tiempos humanos» de una mente «desplegada», pero también «enfriada» («assiderata»): la inocente sencillez de costumbres generosas como la fresca fecundidad de una espontánea producción de poesía.

El tenor de todas maneras "avanzado" de similar posición se puede hallar sobre todo en las aperturas "universalistas" a la comunidad de las experiencias de todos los pueblos en el fatigoso avance de bárbaras formas primigenias a formas poco a poco más «humanas». De ahí la idea extraordinaria de que los americanos, si no hubieran sido obstaculizados, habrían podido desarrollarse hasta la madurez civil, con sus facultades y religiones: una especie de "apocatástasis universal" de la salvación de las naciones. ${ }^{6}$ En esa nota de marcado universalismo se puede hallar uno de los aspectos más vitales del pensamiento "político" viquiano, así como en el

6. Con respecto a esto, he retomado elementos de un ensayo mío recién publicado: «Vico nel Settecento. Vico tra gli illuminismi?», Bollettino del Centro di studi vichiani, XLVIII, 2018, pp. 59-66. 
no consumido problema del nexo de relación-distinción entre "comprensión" y "legitimación" de las formas de dominio. El axiológico "principio del mérito" viquiano -según el cual es justo que a los mejores les corresponda el mando- se articula en una dirección "naturalista" (hay una predisposición natural de algunos sujetos al dominio) y en una dirección superior marcadamente "universalista" (cuando faltan las razones iniciales de legitimación del poder, estas se transfieren a otras figuras históricas que luchan por el poder mismo).

No existe ninguna primacía con respecto a lo "étnico" o "cultural", sino más bien la idea de una tendencia a un desplazamiento continuo de las razones de la legitimación del poder que parece seguir siendo digno de atención. Al pasar de una representación "platónica", "intra-antropológica" del conflicto entre mente y cuerpo, a una comprensiva representación histórica, el horizonte de la «equidad», de la justedad de los "derechos", de lo que les corresponde a los hombres, no es el del pasado: se trata de una exigencia y una presión que, al proceder de una latencia remota, por eso mismo contiene el estímulo hacia el "aún no", el "más allá".

Con eso se ha regresado al ámbito -que considero muy resbaladizo pero imprescindible de las "herencias" de Vico. Tal y como ya dije en otra ocasión, estas -así como aquellas de cualquier otro clásico- se pueden comprender mejor no porque se sustraen a su tiempo, sino más bien porque nos hablan más de su tiempo, tanto cuando se configuran como herencias de alguna manera "perdidas" (por ser dispersas, o por ser consumidas en cuanto "metabolizadas"), como cuando se presentan como herencias que exceden de ese tiempo, es decir, aquellas que los herederos prefieren, producen.

Dentro de estas últimas, el conjunto de las ideas propias de la consideración historicizante de todos los fenómenos de lo real (de hecho autónomamente elaborado en la reflexión de los siglos XIX y XX) puede reaparecer más vivo precisamente si se retoma en su íntegra determinación histórica; así como el atractivo del complejo y vívido estilo narrativo por medio del que ese conjunto está dicho queda abierto aún más para quien logre advertir la consistencia histórica de semejante y peculiar estilo. De la misma manera, el conjunto de ideas relativo a las "facultades ingeniosas" aparece más vital cuanto más se vuelva a leerlo en la extraordinaria capacidad de innovación que poseía en su tiempo, cuando seguía estando vigente (aunque no absolutamente) la perspectiva de un sujeto cognoscitivo y ético atemporal. Ya se ha dicho que lo que presenta un atractivo hoy más "actual" es, tal vez, el conjunto de ideas atinente a una destacada tarea práctica de la filosofía, a una correlativa orientación universalista, y a un llamado a las responsabilidades comunitarias para contrastar los insipientes «orgullos» («borie») de las naciones y de los eruditos y las aptitudes a formas de flanqueo vilmente «delicado» de los hombres.

Sin embargo, sigo desconfiando bastante de las investigaciones de la actualidad, aún más en los tiempos omnívoros de la última contemporaneidad; con más 
razón con respecto a un "clásico", que debería aparecer como lo que resiste tendencialmente "por completo" al paso del tiempo; lo cual es posible solo si de este no se privilegian demasiado los aspectos llegados a ser más apetecibles. De alguna manera la experiencia de interés por la dimensión del viviente-pático que algunos estudiosos han opinado ver en Vico debería ser respetada antes que nada por el lector, por lo menos por el intérprete, que debe enfrentarse con un orgánico "cuerpo de pensamiento".

Una especie de pietas del historiador -que tal vez pueda otorgar un calificativo "ético" a su trabajo (en mi caso, a una curiosidad nunca satisfecha de explorar plúrimos pero específicos horizontes de sentido)- se halla en el intento de "salvar", aunque provisionalmente y en la conciencia de una sospechada constitutiva inanidad, algo de lo que encuentra en los pliegues reclinados o perdidos del pasado, sometido a su incesante y desmedida dispersión. La mayoría de las veces lo que él encuentra o saca a la luz no parece tener nada o poco de lo estrechamente "actual". Sin embargo, quizás tenga no menos derecho de volverse a hacer "presente" con respecto a lo que con mayor fuerza resiste del gran "clásico", como sus "herencias" que nos hablan más, y que obviamente es menester primero que nada traer a la memoria. Desde luego, el mismo "clásico" tiene sus recovecos, sus "páginas" que tienden a desteñirse y a salir de la memoria, a perder su presencia, a distanciarse del "cuerpo" originario del texto. El mismo Vico los tiene. Piénsese en las largas secciones de las "Ciencias nuevas" en las que enumera y reconstruye sus etimologías (en ese caso, sin embargo, está en juego una remota denominación "natural" de las cosas). También el historiador -y aun antes el filólogo- de Vico debe intentar "salvar" como pueda también esas páginas que fueron pensadas como "vivientes" en el texto, tal vez por esto hecho aún más vivo.

[Traducción del italiano por Maria Rosaria Colucciello]

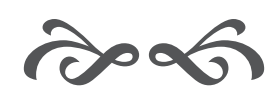

